\title{
Guest editorial: Focused section on advances in soft robotics
}

\author{
Xiaobo Tan ${ }^{1} \cdot$ Kam K. Leang ${ }^{2} \cdot$ Zhouping Yin $^{3}$
}

Received: 7 April 2017/Accepted: 9 April 2017/Published online: 18 April 2017

(C) Springer Singapore 2017

Inspired by biological organisms including worms, octopuses, and starfish, the emerging field of soft robotics aims to create robots with soft structures, sensors, and actuators (Rus and Tolley 2015; Laschi et al. 2012; Brown et al. 2010; Shepherd et al. 2011; Rogers et al. 2010; Park et al. 2010). Compared with traditional "rigid" robots, soft robots can more safely interact with humans. Additionally, their "soft" and compliant bodies enable them to navigate through unstructured, cluttered environments, for example, squeezing through narrow openings and turning tight corners. Potential applications of soft robots include robotic surgery, prosthetics and orthotics, elder care, surveillance, search and rescue, among others. The development of soft robots presents a number of challenges in material synthesis, mathematical modeling, mechanism design, and control, and in recent years has attracted increasing

Xiaobo Tan

xbtan@egr.msu.edu

Kam K. Leang

kam.k.leang@utah.edu

Zhouping Yin

yinzhp@mail.hust.edu.cn

1 Department of Electrical and Computer Engineering, Michigan State University, East Lansing, MI 48824, USA

2 Department of Mechanical Engineering, University of Utah, Salt Lake City, UT 84112, USA

3 School of Mechanical Science and Engineering, Huazhong University of Science and Technology,

Wuhan 430074, Hubei, China attention from researchers. For instance, a soft robot comprised of sensors, actuators, and structures, all with soft, deformable, and compliant characteristics, requires advances in material development and manufacturing technology. Also, it is crucial to have distributed, effective control architecture that requires minimal computing power. Finally, it is of great importance to develop computationally-efficient modeling tools for soft and deformable materials and structures.

The goal of this focused section (FS) is to highlight some of the recent advances made in the field of soft robotics. A total of 13 submissions were received. After a rigorous peer-review process, eight papers were accepted to be included in this FS. Among these, the first two papers focus on novel materials for soft robots, the next three papers explore sensing and actuation mechanisms, and the last three papers examine robotic systems involving soft or flexible elements. A brief description of each paper's contributions follows.

\section{Novel materials for soft robots}

In their paper, Walker et al. introduce a naturally degradable material for pneumatic actuators, which consists of an elastomer, poly(glycerol sebacate), with an additive calcium carbonate (PGS-CaCO3). The authors describe the synthesis of the material, which involves simple steps and only uses inexpensive and non-hazardous chemicals. Mechanical properties of the proposed material and performance of the resulting pneumatic actuators are reported under static, cyclic, and compressive loading conditions. The degradability of the material is also discussed. Such materials will contribute to the development of soft robots that are environmentally friendly and safe for biomedical 
applications, especially for applications where the retrieval of robots might be impossible.

The paper by Fallahi et al. presents a novel ionic electro-active polymer called poly (ethylene-comethacrylic acid) metal composite (EMAMC). Similar to ionic polymer-metal composites (IPMCs), EMAMC is obtained by chemically plating an ionomer and can serve as soft sensors, actuators, and energy harvesters. Different from IPMCs, which typically use Nafion as the base polymer, the proposed approach uses poly (ethylene-comethacrylic acid), which has self-healing properties and is much less expensive than Nafion. The authors describe the procedure for fabricating EMAMC and report the results on the characterization of its actuation and sensing behaviors.

\section{Soft sensing and actuation mechanisms}

Pinto et al. present a new approach to the fabrication of integrated strain sensors for soft pneumatic actuators (SPAs) based on carbon nanotubes (CNTs). The sensors are created with CNT ink through a screen printing process. The sensing principle is based on the resistance change of the CNT sensor under different strains. Silver nanowires are used to create the electrical leads for the sensors. The authors describe the fabrication process for the SPA with integrated CNT sensors and characterize the sensing performance of the sensors when the SPA is actuated to different curvatures.

The next paper by Stalbaum et al. explores an array of ionic polymer-metal composite (IPMC) actuators for generating traveling waves in an artificial wing. Inspired by the wing behavior of a flying fish during descent, such shape modulations could be used to adaptively tune the aerodynamics of a flying robot in response to wind and other conditions. In their paper, the authors describe how the active IPMC wing is constructed and controlled to produce the desired traveling wave patterns.

The paper by Choi et al. describes millimeter-scale micro-robotic leg actuators that integrate thin-film lead zirconate titanate (PZT) piezoelectric actuation with compliant structures formed from parylene-C polymer. Design and modeling of the actuation mechanisms are presented. A hexapod robot prototype incorporating multiple actuators in each leg assembly is fabricated and tested, and the experimental results show close agreement with the model predictions of static and dynamic characteristics. These results reveal advantages of the device in terms of low power requirements relative to payload capacity and large amplitude motion at resonance.

\section{Robotic systems involving soft or flexible elements}

The first paper in this group, authored by $\mathrm{Ye}$, Hou and Chen, deals with a bio-inspired underwater robot motivated by mobile sensing applications. The robot has a pair of flexible pectoral fins and a whale-like fluke, all actuated by IPMC bending actuators, and it is capable of swimming and maneuvering in the horizontal plane. A dynamic model for the robot is developed. Experiments are conducted to characterize the swimming and turning performance of the robot and validate the proposed model.

The next paper by Phamduy et al. also focuses on robotic fish, but their research is motivated by the study of social behavior of live fish. Available robotic fish generally exceed the size of popular fish species for laboratory experiments, and therefore achieving small size for the robotic fish is essential to effective investigation of robotanimal interactions. The authors leverage multi-material 3D printing to create a miniature robotic fish. Thrust and drag of the robot are characterized with several experimental techniques, which are subsequently used to develop reduced-order models and study the motion of the robotic fish.

Finally, Tan and Ren present a flexible robot for endoscope steering, to be used for minimally invasive surgeries. The robot employs multiple spring support units instead of backbones, and it uses pulling and releasing of wires to achieve the movement and maneuvering of the end-effector. Detailed design analysis is presented, and a prototype is developed and tested, which shows the capability of steering an endoscope with five degrees of freedom.

\section{References}

Brown, E., Rodenberg, N., Amend, J., Mozeika, A., Steltz, E., Zakin, M.R., Lipson, H., Jaeger, H.M.: Universal robotic gripper based on the jamming of granular material. Proc. Natl. Acad. Sci. 107(44), 18809-18814 (2010)

Laschi, C., Cianchetti, M., Mazzolai, B., Margheri, L., Follador, M., Dario, P.: Soft robot arm inspired by the octopus. Adv. Robot. 26(7), 709-727 (2012)

Park, Y.L., Majidi, C., Kramer, R., Bérard, P., Wood, R.J.: Hyperelastic pressure sensing with a liquid-embedded elastomer. J. Micromech. Microeng. 20(12), 125029 (2010)

Rogers, J.A., Someya, T., Huang, Y.: Materials and mechanics for stretchable electronics. Science 327(5973), 1603-1607 (2010)

Rus, D., Tolley, M.T.: Design, fabrication and control of soft robots. Nature 521, 467-475 (2015)

Shepherd, F., Ilievski, F., Choi, W., Morin, S.A., Stokes, A.A., Mazzeo, A.D., Chen, X., Wang, M., Whitesides, G.M.: Multigait soft robot. Proc. Natl. Acad. Sci. 108(51), 20400-20403 (2011) 


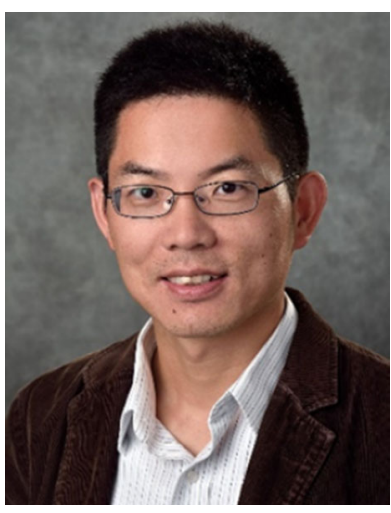

Xiaobo Tan received the B. E. and M. E. degrees in automatic control from Tsinghua University, Beijing, China, in 1995 and 1998, respectively, and the Ph.D. degree in electrical and computer engineering from the University of Maryland, College Park, in 2002. From September 2002 to July 2004, he was a Research Associate with the Institute for Systems Research at the University of Maryland. $\mathrm{He}$ joined the faculty of the Department of Electrical and Computer Engineering at Michigan State University (MSU) in 2004, where he is currently an MSU Foundation Professor. His research interests include modeling and control of systems with hysteresis, electroactive polymer sensors and actuators, soft robotics, and bioinspired underwater robots and their application to environmental sensing. Dr. Tan has served as an Associate Editor/Technical Editor for Automatica, IEEE/ASME Transactions on Mechatronics, and International Journal of Advanced Robotic Systems. He served as the Program Chair of the 2011 International Conference on Advanced Robotics, and the Finance Chair of the 2015 American Control Conference. He has coauthored one book (Biomimetic Robotic Artificial Muscles) and over 200 journal and conference papers, and holds one US patent. He is a Fellow of IEEE, a recipient of NSF CAREER Award (2006), a recipient of MSU Teacher-Scholar Award (2010), and several best paper awards.

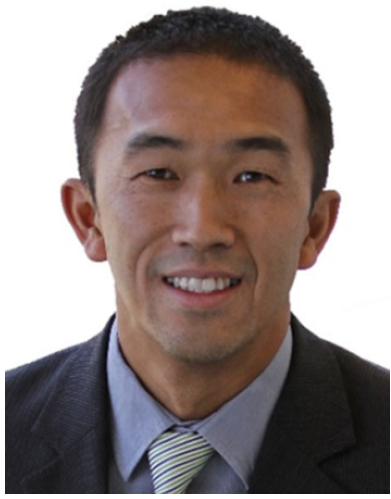

Kam K. Leang received the B.S. and M.S. degrees in mechanical engineering from the University of Utah, Salt Lake City, in 1997 and 1999, respectively, and the Ph.D. degree from the University of Washington, Seattle, in December 2004. He is an Associate Professor in the Mechanical Engineering Department at the University of Utah, where he joined in July 2014 and directs the DARC (Design, Automation, Robotics and Control) Lab. $\mathrm{He}$ is also a member of the University of Utah Robotics Center.
Between 2008 and 2014, he was at the University of Nevada, Reno. While in Nevada, he received the 2014 Nevada System of Higher Education Board of Regent's Rising Researcher of the Year Award. His current research interests focus on three main areas: (1) modeling and control for high-speed nanopositioning and scanning probe microscopy, (2) modeling, control, and manufacutring of electroactive polymers for applications in soft robotics, and (3) design, control, and motion planning of mobile robotic systems, including aerial robotic systems. Dr. Leang has served as an Associate/Technical Editor for IEEE/ASME Transactions on Mechatronics, IEEE Control Systems Magazine, Mechatronics (Elsevier), the International Journal of Intelligent Robotics and Applications (IJIRA), and Frontiers in Mechanical Engineering (Nature Publishing). Also, he has been involved with conference organization and editorship, including the American Control Conference (ACC), IEEE International Conference on Robotics and Automation (ICRA), and IEEE/ASME International Conference on Advanced Intelligent Mechatronics (AIM). He is a member of the ASME and IEEE.

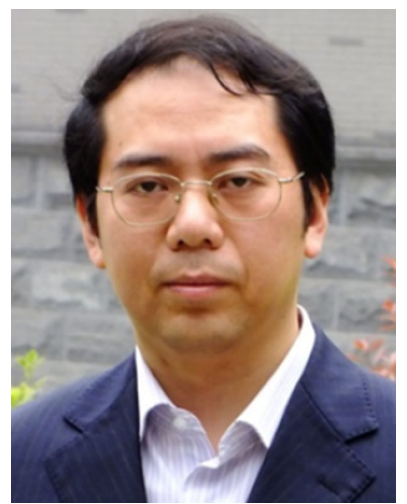

Zhouping Yin received the B.S., M.S. and Ph.D. degrees in mechanical engineering from Huazhong University of Science and Technology (HUST), in 1994, 1996 and 2000, respectively. $\mathrm{He}$ is a Professor in School of Mechanical Science and Engineering, HUST. Since 2005, he has been Vice Head of the State Key Laboratory of Digital Manufacturing Equipment and Technology at HUST. $\mathrm{He}$ was awarded the China National Funds for Distinguished Young Scientists in 2006. He has been a "Cheung Kong" Chair Professor of HUST since 2009. He has been a principal investigator for projects sponsored by National Science Foundation of China (NSFC), National Basic Research Project (973) of China and others. He has coauthored three books and over 100 international journal papers. His research interests include electronic manufacturing equipment and technology, RFID technology and applications, digital manufacturing and applications. 